\title{
The Possible Anti-fibrotic Effect of Artichoke on the Liver and Heart of Bile Duct Ligation Rat Model
}

\author{
Neveen M El-Sherif* and Noha M Issa \\ Department of Anatomy and Embryology, Menoufia University, Egypt \\ Submission: March 08, 2017; Published: March 23, 2017 \\ *Corresponding author: Neveen M El-Sherif, Department of Anatomy and Embryology, Faculty of Medicine, Menoufia University, Egypt, \\ Email: neveensherif@yahoo.com
}

\begin{abstract}
Cirrhosis may be accompanied by attenuated ventricular responsiveness with an increased resting cardiac output; this is termed cirrhotic cardiomyopathy. The artichoke could be considered to have possible anti-diabetic, hepatoprotective and anti-oxidant potential but with little information about the mechanisms underlying its effect. The root contains little amounts of fat and zero cholesterol. In addition to small proportions of minerals and vitamins, also it has small amounts of anti-oxidant vitamins such as vitamin C, vitamin A, vitamin E. These vitamins act with flavonoid compound like carotenes as free radicals scavenger.
\end{abstract}

Abbreviations: BDL: Bile Duct Ligation; ALT: Alanine Transaminase; AST: Aspartate Transaminase; H\&E: Haematoxylin and Eosin; $\alpha$ SMA: $\alpha$ Smooth Muscle Actin; rER: rough Endoplasmic Reticulum; HSCs: Hepatic Stellate Cells; ECM: Extra-Cellular Matrix proteins

\section{Introduction}

The causes of liver fibrosis vary including viral hepatitis, alcohol abuse, drugs, metabolic diseases due to overload of iron or copper, autoimmune attack on hepatocytes or congenital abnormalities can cause chronic hepatic injury [1]. Obstructive jaundice is the condition of bile duct obstruction and reduced bile flow. This cholestasis causes intrahepatic accumulation of reactive oxygen species, oxidative stress and lipid peroxidation which lead to cholestatic liver fibrosis [2]. Beside the oxidative stress, the cholestasis leads to accumulation of toxic hydrophobic bile salts within hepatocytes, causing inflammatory reactions, hepatocytes death, and periductular fibrosis [3]. Bile duct ligation (BDL) could be considered as a good model of biliary cholestasis in animals that induced oxidative damage and fibrosis in rats [4]. Cirrhosis may be accompanied by attenuated ventricular responsiveness with an increased resting cardiac output; this is termed cirrhotic cardiomyopathy [5]. The artichoke could be considered to have possible anti-diabetic, hepatoprotective and anti-oxidant potential but with little information about the mechanisms underlying its effect [6]. The root contains little amounts of fat and zero cholesterol. In addition to small proportions of minerals and vitamins [7], also it has small amounts of anti-oxidant vitamins such as vitamin $\mathrm{C}$, vitamin A, vitamin E. These vitamins act with flavonoid compound like carotenes as free radicals scavenger [8].

\section{Methodology}

Animals

Fifty adult Wistar male albino rats were used in this experiment, each weighting 150-200 grams. Food and water were provided ad libitum and the rats were left for 7 days for acclimatization before use in the Anatomy Department, Faculty of medicine, Menoufia University. All aspects of animal care and treatment were carried out according to the local guidelines of the ethical committee for animal research.

\section{Experimental plan}

The animals were divided into five groups as the following:

Group I (Control): included ten rats were kept without any treatment throughout the experimental period.

Group II (Artichoke treated): included ten rats that received artichoke (Artichoke extract), a product of Western Pharmaceutical industries for International Medical Guide, was available in the form of capsules with the trade name 'Super Artichoke'. Each capsule contained $320 \mathrm{mg}$ of the dried roots of artichoke. The contents of the capsule was withdrawn and dissolved in distilled water and given to the rats in a dose of 25 $\mathrm{mg} / \mathrm{kg}$ once daily for three weeks by gastric tube). 
Group III (Sham operated group): included ten rats to which abdominal incision was made without a bile duct ligation.

Group IV (Bile duct ligated group): included ten rats to which bile duct ligation operation was done. Rats were anesthetized with ketamine and chlorpromazine $(100 \mathrm{mg} / \mathrm{kg}$ ketamine and $0.75 \mathrm{mg} / \mathrm{kg}$ chlorpromazine; ip). Under antiseptic conditions, a mid-line abdominal incision was made, exposing the muscle layers and the linea alba, which were then incised over the length corresponding to the skin incision. The edge of the liver was then raised and the duodenum pulled down to expose the common bile duct, a blunt aneurysm needle was passed under the part of the duct selected, stripping the pancreas away with care and the duct was ligated with prolene thread. The peritoneum and the muscle layers as well as the skin wound were closed with prolene stitches [9].

Group V (Bile duct ligated artichoke treated group): included ten rats to which bile duct ligation operation was done, two weeks after the operation, the super artichoke was given in a dose of $25 \mathrm{mg} / \mathrm{kg}$ once daily for three weeks by gastric tube.

\section{Biochemical study}

At the time of sacrifice, blood samples were collected to measure the serum levels of total bilirubin in $\mathrm{mg} /$ day. Alanine transaminase (ALT) and aspartate transaminase (AST) were also measured and expressed in IU/l. They were measured with routine laboratory methods in the Biochemistry Department, Faculty of Medicine, Menoufia University.

\section{Histological study}

\section{Light microscopic study}

Liver and heart samples were fixed in $10 \%$ formal saline and processed to prepare $5 \mu \mathrm{m}$-thick paraffin sections for:

- Haematoxylin and Eosin (H\&E) stain

- Immunohistochemical stains for liver sections using a rabbit polyclonal antibody against $\alpha$ smooth muscle actin $(\alpha \mathrm{SMA})$ (Abcam, Cambridge, UK), and for heart sections using vimentin antibody (VIM 3B4, mouse monoclonal antibody; Dako Company, Wiesentheid/Bavaria). In brief, the immunohistochemical staining was carried out by means of the avidin biotin-peroxidase complex (Dako Company, Wiesentheid/ Bavaria, Germany, Biotin Blocking System) method following the manufacturer's instructions. Paraffin sections of $5 \mu \mathrm{m}$ were deparaffinized in xylene and rehydrated in a descending series of ethanol. The specimens were subjected to antigen retrieval in a citrate buffered solution ( $\mathrm{pH}$ 6.0) for $10 \mathrm{~min}$ using a microwave. Endogenous peroxidase was eliminated by incubation in $10 \%$ $\mathrm{H} 2 \mathrm{O} 2$ in phosphate-buffered saline (PBS; pH 7.4) for $10 \mathrm{~min}$. After washing, the specimens were blocked in ready-use normal goat serum for $20 \mathrm{~min}$ at room temperature.

\section{Electron microscopic study}

Small liver and heart specimens (1 $\mathrm{mm} 3)$ were prepared for electron microscopic examination. They were fixed in $2.5 \%$ phosphate buffered glutaraldehyde solution ( $\mathrm{pH} 7.4)$ at $4{ }^{\circ} \mathrm{C}$ for $2 \mathrm{~h}$. After washing with phosphate buffer, the specimens were post fixed for $1 \mathrm{~h}$ in $1 \%$ buffered osmium tetroxide solution. Subsequently, the specimens were dehydrated through ascending series of ethanol, treated with propylene oxide, and embedded in epoxy resin. After heat polymerization, the sections were cut using an ultra microtome with a diamond knife and were doublestained with uranyl acetate and lead citrate to be examined by a JOEL (Columbia, South Carolina, USA) electron microscope at the EM unit of Al-Azhar University.

\section{Morphometrical study}

The image analyzer computer system Leica Qwin 500 (Leica Ltd, Cambridge, UK) was used to evaluate the area percentage (area \%) of positive immune reaction for $\alpha$ smooth muscle actin and vimentin. The area percentage and standard measuring frame of a standard area equal to $118476.6 \mathrm{~mm} 2$ were chosen from the parameters measuring 10 readings from five sections from each rat of the randomly chosen five rats in each group. In each randomly chosen field, the sections of the liver and heart were enclosed inside the standard measuring frame; the areas where brown positive immune reaction were seen, were then masked by blue binary color to be measured. These measurements were obtained by total magnification $\times 400$ with the area $\%$ of positive immune reaction.

\section{Statistical analysis}

Data for all groups were expressed as mean $\pm S D(X \pm S D)$. The data obtained from the image analyzer and the biochemical data were subjected to SPSS program version 14. Statistical analysis using the one-way analysis of variance test was carried out.

\section{Results}

Similar morphological results were observed between Group I (Control), Group II (Artichoke treated) and Group III (Sham operated) rats in all the outcomes; therefore, these groups were pooled in one group (Control).

\section{Biochemical parameters}

Rats with Bile duct ligated (BDL) group showed a significant increase in the means of levels of total bilirubin, ALT and AST compared with the control group. Artichoke administration significantly lowered all these parameters in BDL artichoke treated group compared with Bile duct ligated group (Table 1).

Table 1: Significance between means of levels of total bilirubin, alanine aminotransferase and aspartate aminotransferase of different groups.

\begin{tabular}{|c|c|c|c|}
\hline Group & Control & $\begin{array}{c}\text { Bile duct } \\
\text { ligated }\end{array}$ & $\begin{array}{c}\text { BDL artichoke } \\
\text { treated }\end{array}$ \\
\hline Total bilirubin & $0.56 \pm 0.91$ & $2.96 \pm 0.79^{\mathrm{a}}$ & $1.21 \pm 0.02^{\mathrm{b}}$ \\
\hline ALT & $24.52 \pm 3.91$ & $132.29 \pm 3.53^{\mathrm{a}}$ & $61.72 \pm 3.25^{\mathrm{b}}$ \\
\hline AST & $22.37 \pm 1.79$ & $122.21 \pm 3.90^{\mathrm{a}}$ & $47.68 \pm 1.91^{\mathrm{b}}$ \\
\hline
\end{tabular}




\section{Anatomy Physiology \& Biochemistry International Journal}

ALT, alanine aminotransferase; AST, aspartate aminotransferase.

a. Significant difference of Bile duct ligated group compared with control

b. Significant difference of BDL artichoke treated group compared with group Bile duct ligated group

\section{Light microscopic examination of the liver}

In the control group, H\&E-stained liver sections showed the normal architecture of the classic hepatic lobules, with plates of polygonal hepatocytes radiating from the central veins and separated by blood sinusoids. The hepatocytes revealed an acidophilic cytoplasm and rounded vesicular nuclei. The portal tract containing branches of portal vein, hepatic artery, and bile duct was observed (Figure 1).

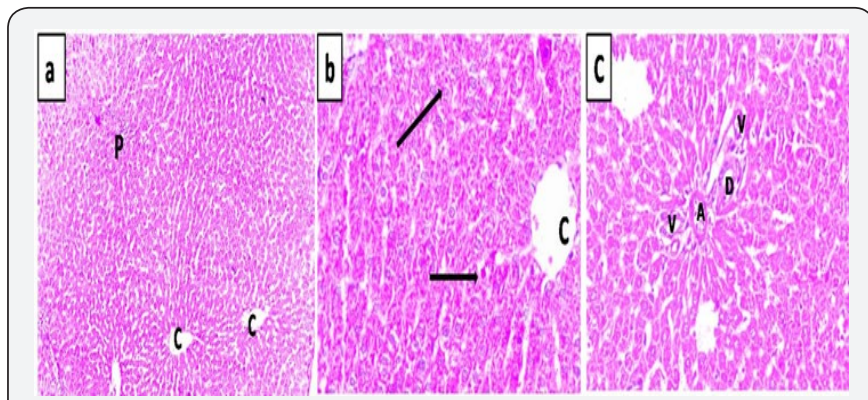

Figure 1: A photomicrograph of a liver section from the control group showing (a) classical hepatic lobule in the form of hepatocytes radiating from the central vein $(\mathrm{C})$ to the periphery of the lobule and portal tracts at the periphery $(P)$; and $(b)$ hepatocytes with acidophilic granular cytoplasm and central vesicular nuclei (arrows). Hepatocytes are separated by narrow blood sinusoids. (c) a portal tract containing tributaries of portal vein $(V)$, branches of hepatic artery $(A)$ and bile duct $(D)$. H\&E, (a): $\times 100,(b)$ and (c): $\times 400$.

Bile duct ligated sections revealed swollen hepatocytes with light foamy cytoplasm that was filled with vacuoles. Many hepatocytes showed ghost-like appearance with loss of nuclei. The central veins were congested. The portal tract showed apparent dilatation and congestion of the portal vessels and proliferation of bile ducts (Figure 2).

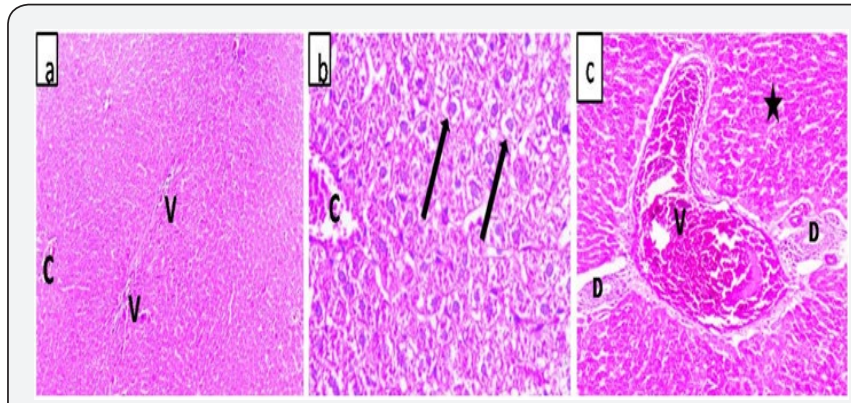

Figure 2: A photomicrograph of a liver section from bile duct ligated group showing (a) congested central vein (C) and portal vessels $(\mathrm{V})$; and (b) swollen hepatocytes with vacuolated cytoplasm (arrows). (c) Hepatocytes with ghost-like appearance with loss of nuclei in many of them (star). Expanded portal tract with dilated congested portal vein $(\mathrm{V})$ and proliferated bile ducts (D) were obviously noted. H\&E, (a): $\times 100,(b)$ and (c): $\times 400$. 


\section{Anatomy Physiology \& Biochemistry International Journal}

Mitochondria were rounded or oval and uniformly distributed Cisternae of rough endoplasmic reticulum (rER) were detected. The hepatocytes were separated by narrow bile canaliculi with characteristic microvilli projecting into the lumen (Figures 5 \& 6).

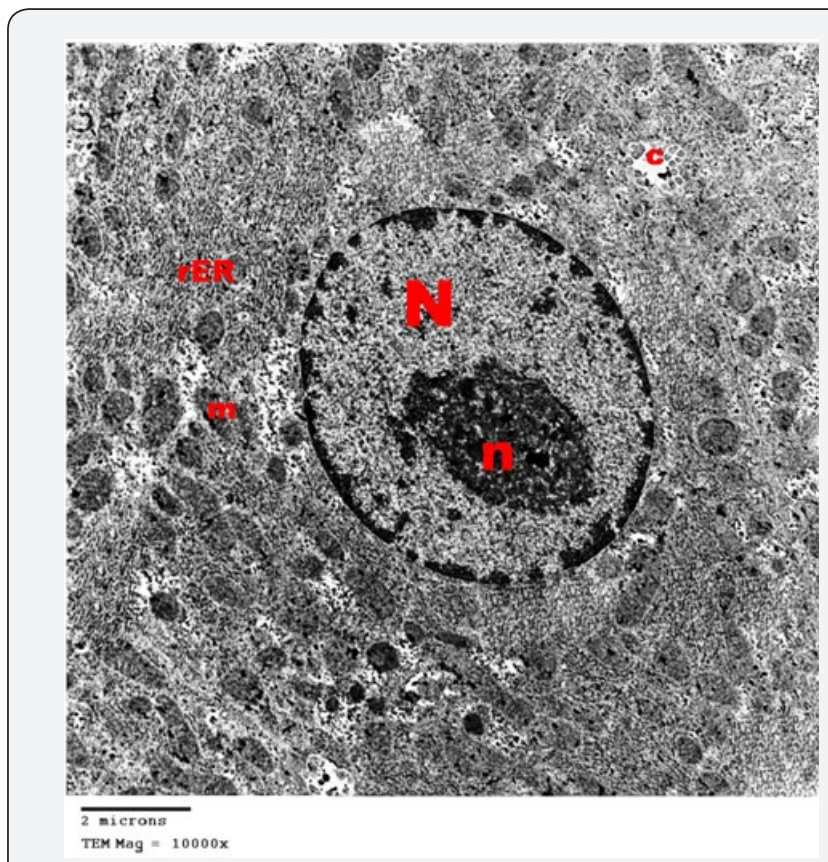

Figure 5: An electron micrograph of control rat liver showing the cytoplasm of the hepatocyte containing rounded euchromatic nucleus $(\mathrm{N})$ with prominent nucleolus $(\mathrm{n})$, oval mitochondria $(\mathrm{m})$ and rough endoplasmic reticulum (rER). Notice the presence of bile canaliculi (c) between hepatocytes. TEM $\times 10000$.

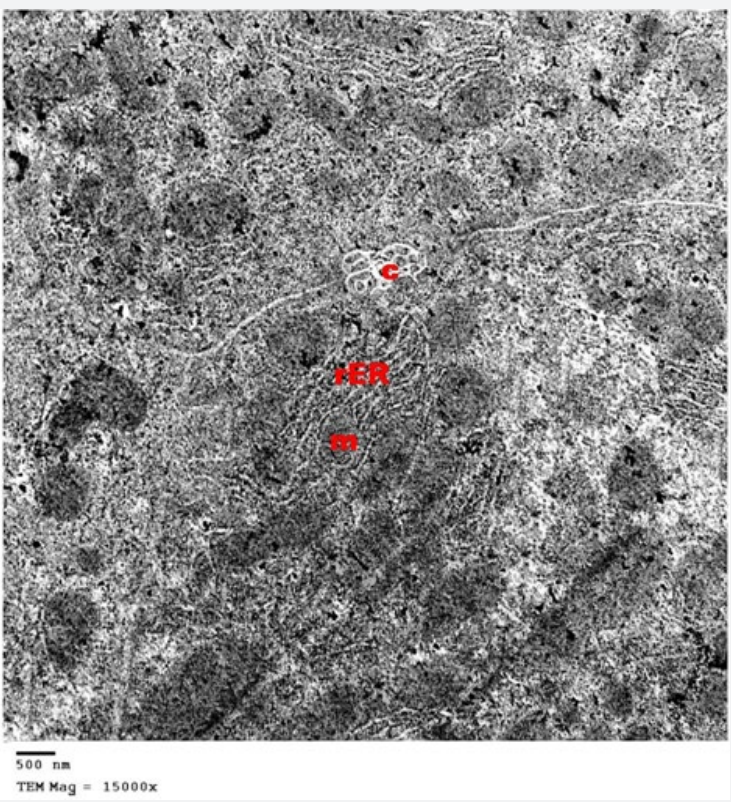

Figure 6: An electron micrograph of the rat liver of control group showing portions of two hepatocytes enclosing a bile canaliculus between them with characteristic microvilli (c). The cytoplasm of the hepatocyte contains rounded or oval mitochondria $(\mathrm{m})$ and cisternae of (rER). TEM $\times 15000$.
Ultrathin sections of the liver of the bile duct dilated group showed that most of the hepatocytes had lost their organelles and the cytoplasm appeared rarified (electron lucent, with loss of a majority of its organelles). Some hepatocytes contained vesicular nuclei with clumped dense chromatin (Figure 7). Some nuclei appeared irregular in shape while others appeared darkly stained and shrinked. In many hepatocytes, the cytoplasm appeared vacuolated and contained many collagen fibers and lipid droplets (Figures 8 \& 9). Dilated bile canaliculi were clearly noted in many hepatocytes (Figure 9). Liver sections of bile duct ligated artichoke treated group showed nearly normal hepatocytes however, some areas of vacuolated cytoplasm, few lipid droplets and slightly dilated bile canaliculi were present (Figure 10).

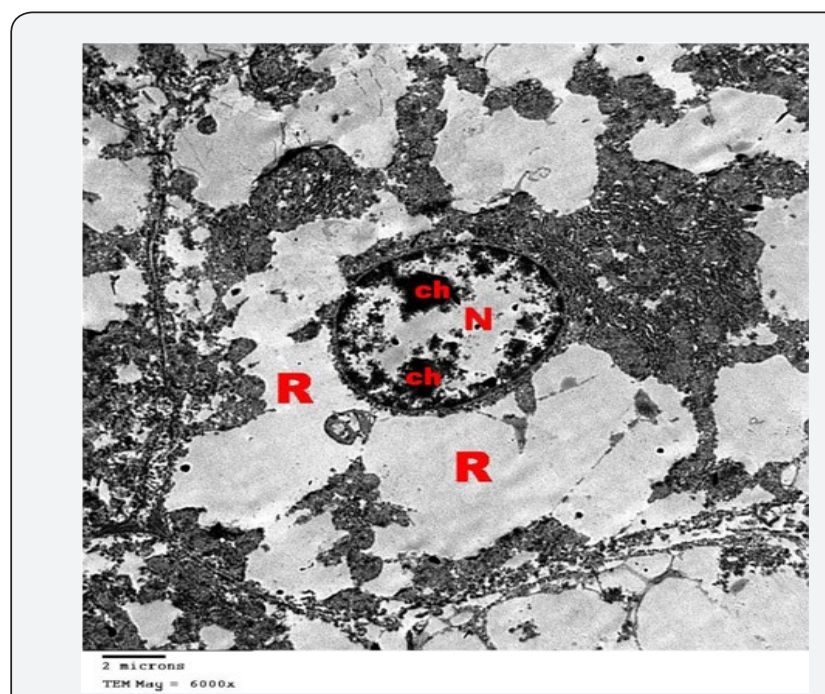

Figure 7: An electron micrograph of the rat liver of bile duct ligated group showing hepatocyte with vesicular nucleus $(\mathrm{N})$ and clumped chromatin (ch). The cytoplasm appeared rarefied (R) with destroyed organelles. TEM $\times 6000$.

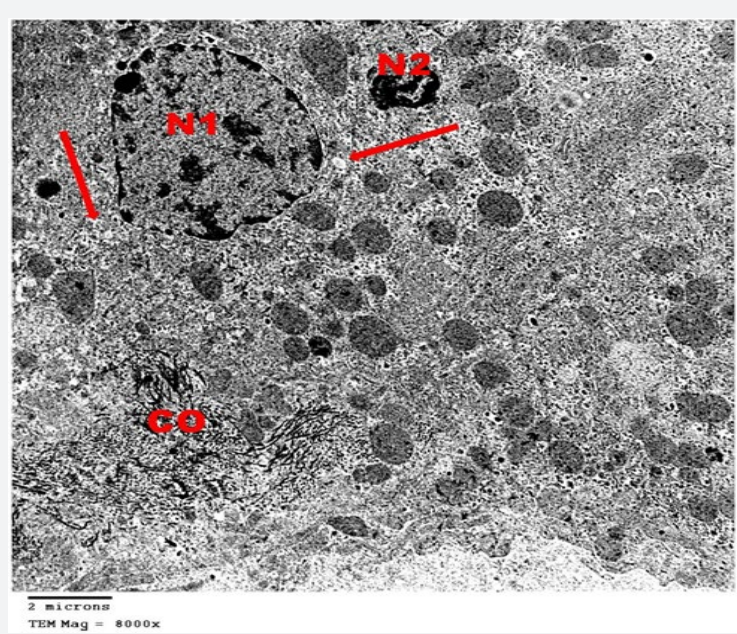

Figure 8: An electron micrograph of the rat liver of bile duct ligated group showing binucleated hepatocyte. One nucleus appeared irregular in shape (N1) while the other appeared darkly stained and shrinked (N2). The cytoplasm appeared vacuolated (arrows) and contained many collagen fibers (co). TEM $\times 8000$. 


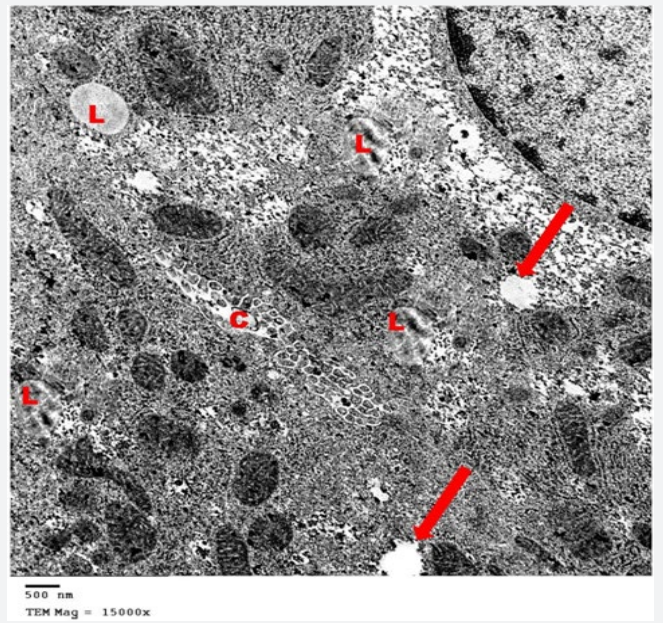

Figure 9: An electron micrograph of the rat liver of bile duct ligated group showing parts of two hepatocytes enclosing dilated bile canaliculus (C) between them. The cytoplasm appeared vacuolated (arrows) and contained multiple lipid droplets (L). TEM $\times 15000$.

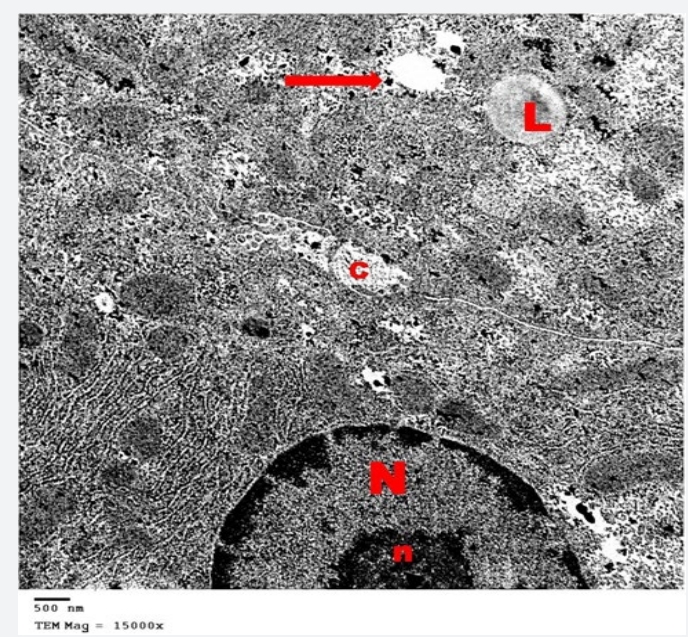

Figure 10: An electron micrograph of the rat liver of bile duct ligated artichoke treated group showing nearly normal architecture of hepatocytes. The cytoplasm contained rounded euchromatic nucleus $(\mathrm{N})$ with prominent nucleolus $(\mathrm{n})$. However, some areas of vacuolated cytoplasm (arrow), few lipid droplets (L) and slightly dilated bile canaliculus (C) were still present in this group. TEM $\times 15000$.

\section{Light microscopic examination of the heart}

Light microscopic examination of the myocardium of the control rats showed that it was formed of organized branching and anastomosing muscle fibers. Cardiomyocytes had acidophilic sarcoplasm and central vesicular nuclei. Capillaries and fibroblasts were seen in the connective tissue endomysium between the cardiac muscle fibers (Figure 11). Myocardial sections from bile duct ligated group showed that cardiomyocytes were separated from each other by wide intercellular spaces. Some of the cardiac muscle fibers attained increased number of fibroblasts. The nuclei of most of the cardiac muscle fibers were central vesicular nuclei, but some appeared small and deeply stained (pyknotic). Many fibers exhibited cytoplasmic lysis. Congested blood capillaries and extravasated red blood cells were clearly noted (Figure 12).

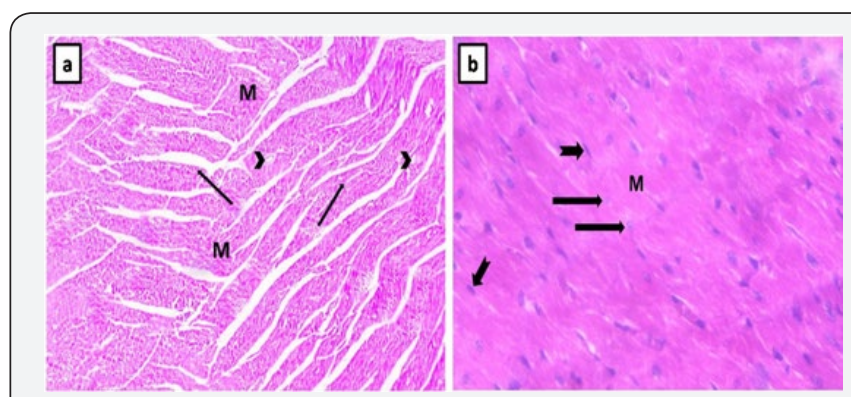

Figure 11: A photomicrograph of a myocardial section from a control rat. It showed organized cardiac muscle fibers ( $M$, a and b) that exhibit acidophilic sarcoplasm and vesicular nuclei (arrows, a and b). Dark nuclei of fibroblasts (notched arrows, b) were seen within the endomysium between the muscle fibers. Note the presence of blood vessels (arrow head, a) between the myocytes. H\&E, (a): × 100, (b): × 400 .

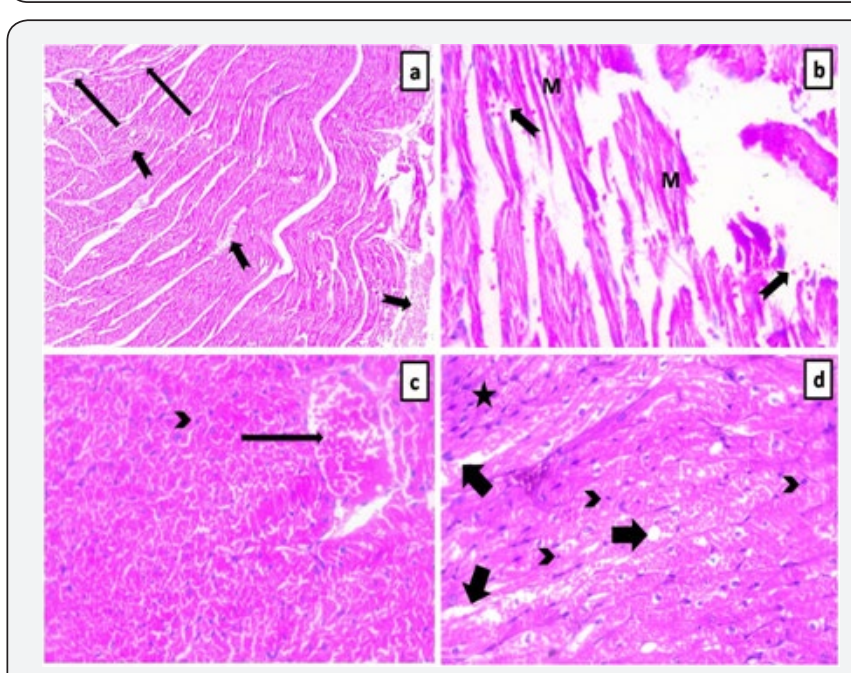

Figure 12: A photomicrograph of a myocardial section from bile duct ligated group showing disorganized disrupted and widely separated muscle fibers $(M, b)$. Some areas showed increased number of fibroblasts (star, d) between the muscle fibers. Cardiac muscle fibers showed the presence of pyknotic nuclei (arrow head, c and d). Some fibers exhibited cytoplasmic lysis (thick arrows, d). Congested dilated blood capillaries (arrows, a and $c$ ) and extravasated red blood cells (notched arrows, a and b) were seen. H\&E, (a): $\times 100,(b-d): \times 400$.

Cardiomyocytes from bile duct ligated artichoke treated group still exhibited pyknotic nuclei and cytoplasmiclysis. Blood capillaries appeared normal however, few of them still congested (Figure 13). Sections of control rat showed that immunohistochemical stain for vimentin revealed brown positive immune reactions for vimentin in the wall of the blood capillaries and some interstitial cells, whereas the cardiac muscle appeared negative in reaction to vimentin. In bile duct ligated sections, vimentin revealed extensive strong brown positive immune reactions in the walls of blood capillaries, and in some interstitial cells. Bile duct ligated artichoke treated group showed a significant decrease in area \% of vimentin immunostaining as compared with bile duct ligated sections (Figure 14). 


\section{Anatomy Physiology \& Biochemistry International Journal}

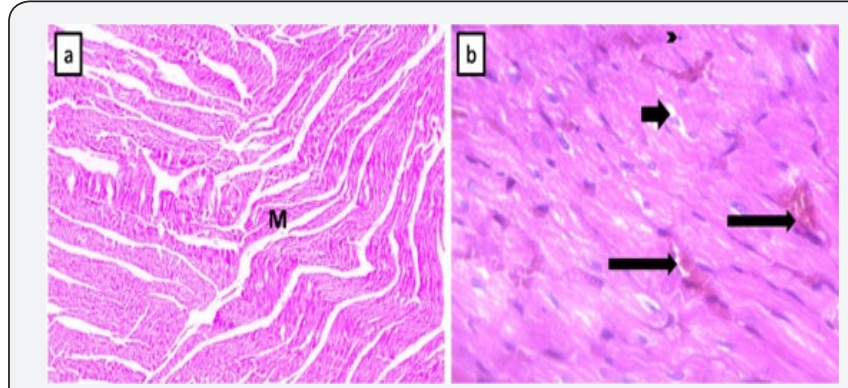

Figure 13: A photomicrograph of a myocardial section from bile duct ligated artichoke treated group showing most of cardiac myocytes appearing normal (M, a). Few muscle fibers exhibited pyknotic nuclei (arrow head, b) and cytoplasmic lysis (thick arrow, b). Few blood capillaries were still congested (arrows, b). H\&E, (a): $\times 100,(b): \times 400$.

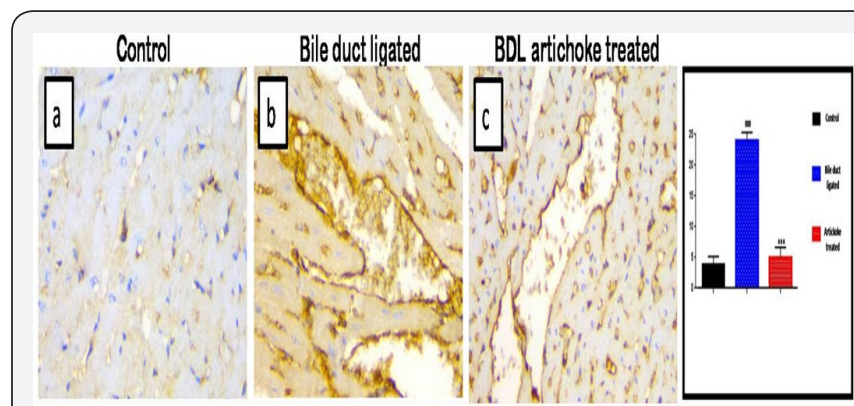

Figure 14: Representative expression of vimentin immunostaining of all experimental groups. Cardiac sections of control rat showed weak positive immunostaining in the wall of blood capillaries, cardiac muscle fibers and interstitial cells (a). Bile duct ligated sections showed a significant up-regulation in vimentin positive reaction especially in the wall of blood capillaries and interstitial cells (b) as compared with control sections. Bile duct ligated artichoke treated group showed a significant downregulation in the activity of vimentin immunostaining (c). ${ }^{000} \mathrm{p}<$ 0.001 , compared to the control group; and ${ }^{* *} p<0.05$ compared to the bile duct ligated group. Vimentin immunostaining, $\times 400$.

\section{Electron microscopic examination of the heart}

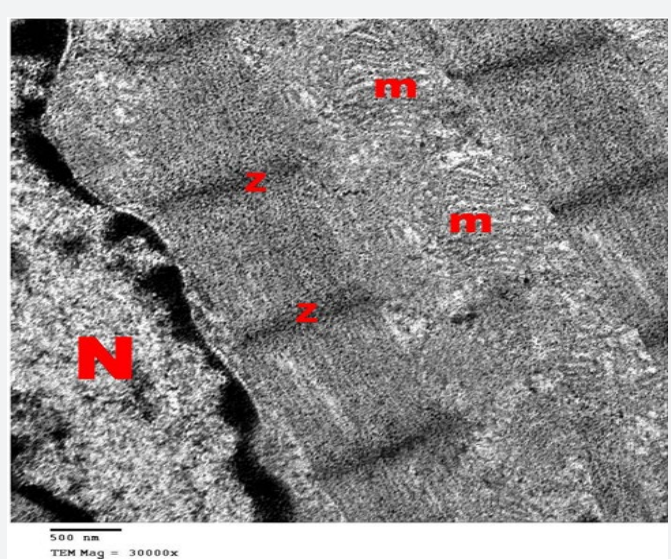

Figure 15: An electron micrograph of control rat showing longitudinally cut cardiac muscle fibers. Oval euchromatic nucleus is seen in one fiber $(\mathrm{N})$. Regularly arranged myofibrils appeared as parallel longitudinal cylinders between successive $Z$ lines bound the sarcomere $(Z)$. Rows of mitochondria $(m)$ are seen in between the myofibrils. TEM $\times 30000$.
Ultra structurally, the myofibers of the control group appeared as longitudinal cylinders containing oval euchromatic nuclei with narrow interfiber spaces. Myofibers were formed of adjacent cardiac muscle cells joined together by step like intercalated discs. Myofibrils are regularly arranged between successive $\mathrm{Z}$ lines in the sarcomeres. Mitochondria with closely packed cristae were detected in between the myofibrils (Figures $15 \& 16)$.

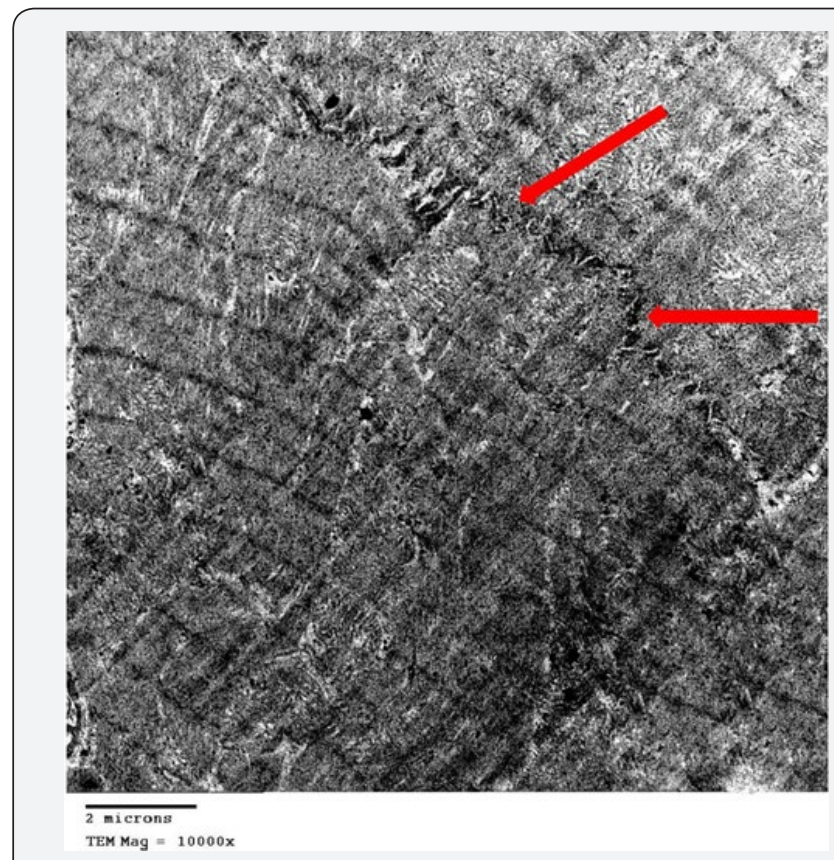

Figure 16: An electron micrograph of control rat showing step like intercalated discs (arrows) between adjacent cardiac muscle fibers. TEM $\times 10000$.

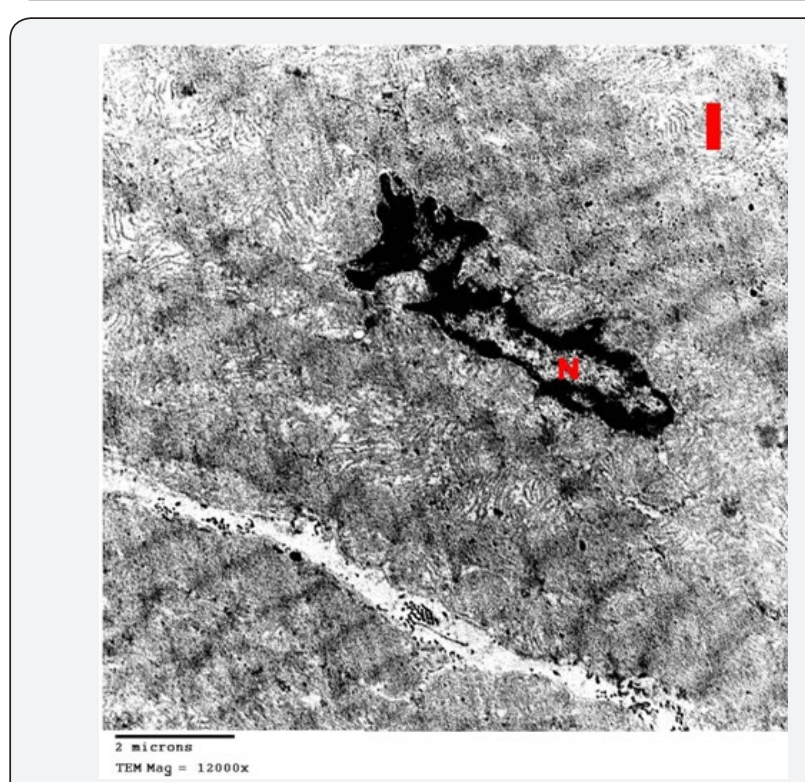

Figure 17: An electron micrograph of rat myocardium of bile duct ligated group showing loss of the normal alignment of cardiac myofibrils that are separated by wide interfiber space (I). Note a muscle fiber which has irregular nucleus with clumping and margination of the heterochromatin (N). TEM $\times 12000$. 


\section{Anatomy Physiology \& Biochemistry International Journal}

Myocardial sections from bile duct ligated group showed focal disruption of myofilaments with irregular nuclei and severely vacuolated cytoplasm. Some of the mitochondria showed disrupted cristae. There was widening of the interstitial space between the cardiac muscle fibers denoting edema. A large number of collagen fibers were found near congested blood capillaries. Widening of the intercalated discs was also noted (Figures 17-20). An electron microscopic examination of myocardium of bile duct ligated artichoke treated group showed almost normal appearance of the cardiac muscle fibers however, widening of interfiber space was still present (Figure 21).

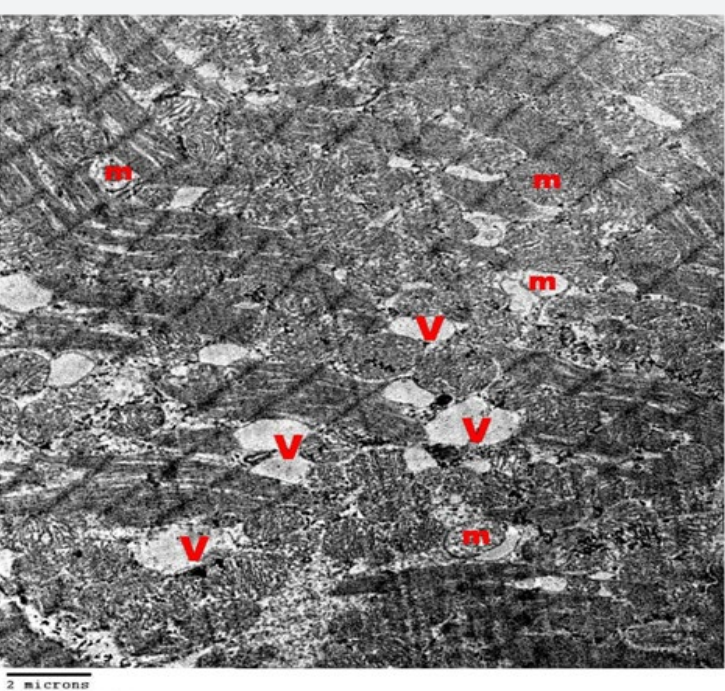

2 microns
TEM Mag $=8000 x$

Figure 18: An electron micrograph of rat myocardium of bile duct ligated group showing disintegrated myofibrils. Many cardiomyocytes were vacuolated and disorganized (V). The mitochondria appeared swollen, degenerated with loss of their cristae $(\mathrm{m})$. TEM $\times 8000$.

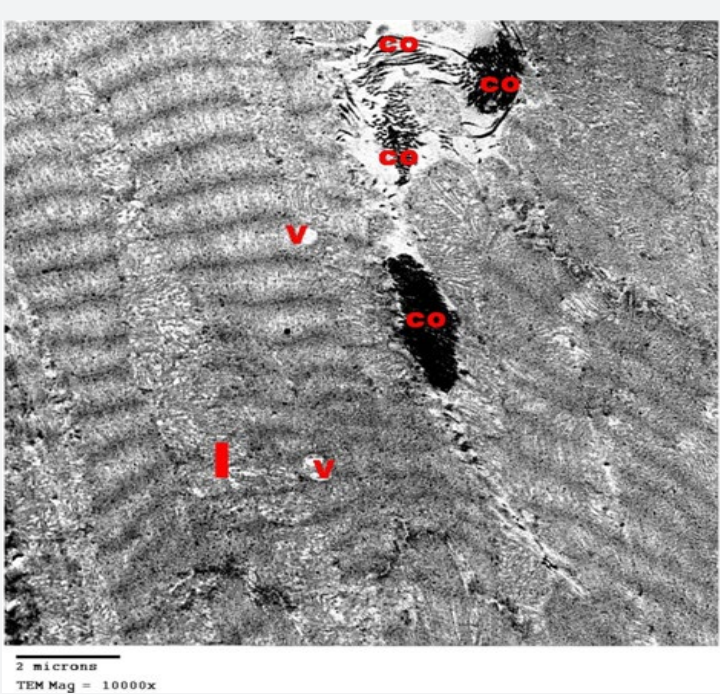

Figure 19: An electron micrograph of rat myocardium of bile duct ligated group showing vacuolated myofibrils (V) that are separated by wide interfiber space (I). Note the presence of a large amount of collagen fibers (CO) in the wide interfiber space. TEM $\times 10000$. 
is a model for chronic cholestasis and cirrhotic cardiomyopathy, significant increase of serum total bilirubin, ALT and AST enzymes in bile duct ligated group emphasis on the occurrence of cholestasis and liver injury in our study. As these biochemical parameters have been reported to be sensitive indicators of liver injury [1]. This cholestasis leads to intrahepatic accumulation of bile acids and toxins with progression of liver pathology, including hepatocellular injury and fibrosis [2]. The liver sections of bile duct ligated group showed many changes included expansion of the portal tract with congested central veins and portal veins; vacuolated and ghost like hepatocytes, these changes were attributed by other authors to bile acid-induced oxidative stress and lipid peroxidation [9]. This oxidative imbalance is due to decreased level of the antioxidant enzymes (GST, SOD and CAT) activities in hepatic tissue that may be attributable to mitochondrial toxicity induced by intrahepatocyte concentration of biliary acids [10].

Ultrathin sections of the liver of the bile duct dilated group showed rarified cytoplasm of most hepatocytes which lost their organelles. This was described as a severe form of oxidative stress which leads to rupture of the cell membrane and loss of the organelles [11]. The nuclear shrinkage, its membrane irregularities and clumping of its chromatin are considered as different stages of apoptosis which results from the difference of DNA sensitivity to oxidative stress together with diminished ability for DNA repair [12]. Also cytoplasmic vacuolation and intracellular accumulation of lipid droplets results from mitochondrial toxicity and dysfunction [13]. The dilated bile canaliculi were clearly noted in many hepatocytes which confirmed the intrahepatic cholestasis resulting from the bile duct ligation.

In accordance with our study biliary proliferation may be a compensatory performance for the damage of cirrhosis [14]. Alpha smooth muscle actin is a reliable immunohistochemical marker for detection of activated hepatic stellate cells (HSCs). In liver cirrhosis, the stellate cells activity is correlated with the degree of fibrosis and necro-inflammatory activity [15]. This explained the up regulation of this marker in the liver slides of bile duct ligated group. Injury of hepatocytes results in the stimulation of Kupffer cells which released some factors lead to activation of HSCs and their transformation into myofibroblastlike cells. Chronically activated HSCs produce large amounts of extracellular matrix proteins (ECM) and enhance fibrosis [16]. The ultrathin sections of the liver of bile duct ligated group showed many collagen fibers which emphasis on the occurrence of fibrosis after the ligation of the bile duct.

The liver sections of bile duct ligated artichoke treated group showed the possible antifibrotic effect of artichoke, through the protection of portal tract components and the hepatocytes together with the down regulation of smooth muscle actin. This in accordance with other authors who stated that artichoke treatment showed promising hepatoprotective effect against CCl4-induced fibrosis via modulation of apoptotic signaling and fibrogenic activity [1]. The ultrathin sections also showed nearly normal hepatocytes however, some areas of vacuolated cytoplasm, few lipid droplets and slightly dilated bile canaliculi but without the occurrence of fibrosis.

Hepatic cirrhosis results in cardiomyopathy which named cirrhotic cardiomyopathy, its histological changes includes myocardial fibrosis, subendocardial edema, and nuclear and cytoplasmic vacuolation of cardiomyocytes [17]. This is in accordance with our results, as the heart sections of bile duct ligated group showed disorganized disrupted and widely separated muscle fibers with increased number of fibroblasts between the muscle fibers, which showed pyknotic nuclei and cytoplasmic lysis; moreover, congested dilated blood capillaries and extravasated red blood cells also present between the degenerated muscle fibers. These changes were explained by hyperdynamic circulation. In which, the heart is overloaded by the persistent increase in cardiac output, associated with the expansion of the circulating blood volume resulting in high cardiac output heart failure [18].

The ultrathin sections of the heart of bile duct ligated group showed focal disruption of myofilaments with irregular nuclei, severely vacuolated cytoplasm and widening of the interstitial space between the cardiac muscle fibers denoting edema. Together with the congested blood capillaries emphasis on the occurrence of high cardiac output heart failure.The presence of large number of collagen fibers supports the occurrence of the fibrosis. Beside the hepatoprotective role of the artichoke, its antifibrotic effect extends to the heart to decrease the oedema, extravasation and degeneration. This appeared precisely in the cardiac ultrathin sections of bile duct ligated artichoke treated group which appeared nearly normal and devoid from the collagen fibers. The mesenchymal marker vimentin identified the fibroblasts based on their spindle shape, the proliferation and activation of the fibroblast is necessary for fibrosis [19]. This explained the up regulation of the vimentin marker in the heart sections of bile duct ligated group and its down regulation in bile duct ligated artichoke treated group.

\section{Conclusion}

We concluded that the intrahepatic cholestasis induced by ligation of the bile duct might lead to hepatic cirrhosis, cirrhotic cardiomyopathy and high cardiac output heart failure. Artichoke could be considered as a novel antifibrotic that protect the liver and the brain of bile duct ligated rat model to great extent.

\section{References}

1. Nabil M, Maiiada H, Ahmed W, Marwa E (2015) Jerusalem artichoke attenuates experimental hepatic fibrosis via modulation of apoptotic signaling and fibrogenic activity. Biochem and Biotech Res 3(3): 43-50.

2. Sohair R, Ayman S (2015) Holoturia arenicola extract modulates bile duct ligation-induced oxidative stress in rat kidney. Int J Clin Exp Pathol 8(2): 1649-1657.

3. Sohair R (2015) Anti-fibrotic effect of Holothuria arenicola extract against bile duct ligation in rats. BMC Complem and Altern Medicine 5: 14 . 
4. Thakare S, Jain H, Patil S, Upadhyay UM (2009) Hepatoprotective effect of Cocculushirsutuson bile duct ligation induced liver fibrosis in Albino Wistarrats. Bangladesh J Pharmacol 4(2): 126-30.

5. Gaskari SA, Liu H, Moezi L, Li Y, Baik SK, et al. (2005) Role of endocannabinoids in the pathogenesis of cirrhotic cardiomyopathy in bile duct-ligated rats. British JPharma 146(3): 315-323.

6. Kim H, Han G (2013) Hypoglycemic and hepatoprotective effects ofjerusalem artichoke extracts on streptozotocin-induced diabeticrats. Food Sci Biotechnol 22(4): 1121-1124.

7. Slavin J (1997) Gut feelings Prebiotic food products play a growing role in good intestinal health. Food-Processing 58: 67-70.

8. El-Houfi A (2005) Technological and biological uses of Jerusalem artichoke powder and resistant starch. Ann Agric Sci 43: 279-291.

9. Han JM, Kim HG, Choi MK, Lee JS, Park HJ, et al. (2012) Aqueous extract of Artemisia iwayomogi Kitamura attenuates cholestatic liver fibrosis in a rat model of bile duct ligation. Food Chem Toxicol 50(10): 35053513.

10. Sanzgiri UY, Srivatsan V, Muralidhara S, Dallas CE, Bruckner JV (1997) Uptake, distribution, and elimination of carbon tetrachloride in rat tissues following inhalation and ingestion exposures. Toxicol Appl Pharmacol 143(1): 120-129.

11. Cogger VC, Mross PE, Hosie MJ, Ansselin AD, McLean AJ, et al. (2001) The effect of acute oxidative stress on the ultrastructure of the perfused rat liver. Pharmacol Toxicol 89(6): 306-311.
12. Hamilton ML, Van Remmen H, Drake JA, Yang H, Guo ZM, et al. (2001) Doesoxidative damage to DNA increase with age? Proc Natl Acad Sci USA 98(18): 10469-10474.

13. Hemmat H Ghafeer, Dalia A El-Gamal, Safaa A Abdel-Maksoud, Fatma Y Abdel Motagally (2009) Evaluation of the Role of Garlic on the Structure of Liver and Gastric Mucosa in Aged Male Albino Rat: A Histological and Histochemical Study. Egypt J Histol 32(1): 129-144.

14. Zhiqiang Z, Zhennan L, Chen Z, Jingjing Z, Yi Z, et al. (2015) Angiogenesis and proliferation of bile duct enhances ischemic tolerance in rats with cirrhosis. Int J Clin Exp Med 8(8): 12086-12095

15. Ionescu AG, Streba LA, Vere CC, Ciurea ME, Streba CT, et al. (2013) Histopathological and immunohistochemical study of hepatic stellate cells in patients with viral C chronic liver disease. Rom J Morphol Embryol 54(4): 983-991.

16. Guo X, Liang B, Wang X, Fan F, Jin J, et al. (2013) Glycyrrhizic acid attenuates CCl4-induced hepatocyte apoptosis in rats via a mediated pathway. World J Gastroenterol 19(24): 3781-3791.

17. Soon K, Tamer R, Samuel S (2007) Cirrhotic cardiomyopathy. Orphanet J Rare Dis 2: 15 .

18. Ma Z, Lee SS (1996) Cirrhotic cardiomyopathy: getting to the heart of the matter. Hepatology 24(2): 451-459.

19. Tracy G, Aster L, Meera R, Seena C, Julie R, et al. (2008) An Immunohistochemical Method for Identifying Fibroblasts in Formalinfixed, Paraffin-embedded Tissue. J Histochem Cytochem 56(4): 347358.

\begin{tabular}{|l|}
\hline \multicolumn{1}{|c|}{ Your next submission with Juniper Publishers } \\
will reach you the below assets \\
- Quality Editorial service \\
- Swift Peer Review \\
- Reprints availability \\
- E-prints Service \\
- Manuscript Podcast for convenient understanding \\
- Global attainment for your research \\
- Manuscript accessibility in different formats \\
( Pdf, E-pub, Full Text, Audio) \\
- Unceasing customer service \\
Track the below URL for one-step submission \\
https://juniperpublishers.com/online-submission.php \\
\hline
\end{tabular}

